\section{New paradigms in the treatment of wet AMD: the impact of anti-VEGF therapy}

G Menon ${ }^{1}$ and $G$ Walters ${ }^{2}$

\begin{abstract}
The incidence of age-related macular degeneration (AMD) in the United Kingdom is increasing with the ageing population. The wet form of this progressive and potentially blinding disease can develop very rapidly and lead to severe loss of central vision and reduction in quality of life, sometimes in just a matter of weeks. Recent advances in the treatment of wet AMD with the licensing of anti-vascular endothelial growth factor therapies, coupled with current guidance from the Scottish Medicines Consortium and the National Institute of Health and Clinical Excellence have led to a subsequent increase in workload at AMD clinics due to the increased number of patients now eligible for treatment. In addition, the Royal College of

the macula that results in a progressive loss of central vision. Although some peripheral vision is maintained, the ability to read, drive and recognise faces is affected and rapid vision loss may occur. ${ }^{1,2}$ In the United Kingdom, AMD accounts for more than half of all registered blindness and partial sight registrations. ${ }^{3}$ Wet (neovascular) and dry (atrophic) forms of AMD differ in their aetiology and presentation. Wet AMD is the most aggressive and is characterised by new growth of abnormal blood vessels from the choriocapillaris (choroidal neovascularisation; CNV), which may be accompanied by haemorrhage and scarring of the macula. ${ }^{1,4}$ Bleeding and fluid leakage frequently occur, leading to very rapid loss of detailed central vision, often within weeks. 5,6
\end{abstract} Ophthalmologists recommend a 2-week diagnosis to treatment schedule due to the aggressive nature of the disease. The role of the retinal specialist is thus changing, and business management skills are becoming increasingly necessary to obtain the necessary resources to implement the guidance. Through prior experience and formal external evaluation of services at Frimley Park and Harrogate District Hospitals, a number of critical success factors have been developed for optimising treatment pathways in efficient wet AMD clinics.

Eye (2009) 23, S1-S7; doi:10.1038/eye.2009.13

Keywords: age-related macular degeneration; anti-VEGF therapy; pegaptanib; commissioning; cost effectiveness; photodynamic therapy

\section{Introduction}

Age-related macular degeneration (AMD) is a serious degenerative eye disease causing photoreceptor dysfunction and deterioration of
Current guidance for the management of wet AMD

The management of individual patients with wet AMD has historically been dependent on the type of wet AMD lesion(s) present and their location in relation to the fovea. In September 2003, the National Institute for Health and Clinical Excellence (NICE) published guidance on photodynamic therapy (PDT) in wet AMD. ${ }^{7}$ It recommended PDT with verteporfin (vPDT) for the treatment of wet AMD in patients with a confirmed diagnosis of classic subfoveal CNV with no sign of occult lesions. Use of vPDT in patients with predominantly classic $\mathrm{CNV}$ is recommended only as part of ongoing or new clinical studies. ${ }^{7}$ On the basis of this guidance, it has been estimated that only approximately $30 \%$ of patients with wet AMD were eligible for vPDT treatment. ${ }^{8}$

More recently, large randomised controlled clinical trials have unequivocally showed clinical efficacy for anti-vascular endothelial
${ }^{1}$ Consultant

Ophthalmologist, Frimley Eye Unit, Frimley Park Hospital, Frimley, UK

${ }^{2}$ Consultant Ophthalmologist, Harrogate District, Harrogate, UK

Correspondence: G Walters, Consultant

Ophthalmologist, Harrogate District Hospital, Lancaster Park Road, Harrogate HG2 7SX, UK.

Harrogate District Hospital Tel: 01423 885959; Fax: 01423555806. Frimley Park Hospital Tel: 01276 604604; Fax: 01483604274. E-mail: gavin.walters@ hdft.nhs.uk 
growth factor (VEGF) therapy in preventing visual loss in people diagnosed with subfoveal wet AMD, irrespective of lesion type which have led to the licensing of two anti-VEGF treatments, ranibizumab (Lucentis ${ }^{\mathbb{\nabla}} \mathbf{\text { }}$ ) and pegaptanib (Macugen ${ }^{\circledR}$ ). ${ }^{9-12}$ Current guidance from NICE and the SMC recommending ranibizumab (Lucentis) throughout the $\mathrm{UK}^{13,14}$ and pegaptanib (Macugen) for a smaller subset of patients in Scotland, ${ }^{15}$ has meant that many more patients with wet AMD are now eligible for treatment.

The Royal College of Ophthalmologists (RCOphth) has also published guidance on commissioning AMD services and also on commencing, continuing, and discontinuing treatment with ranibizumab. ${ }^{8,16}$

\section{Implications of current guidance}

As anti-VEGF drugs can be used to treat all lesion types, a considerable increase in patient numbers is inevitable, as the number of wet AMD patients eligible for treatment increases from 30 to $45 \%$ to approaching $100 \%$ (dependent on meeting NICE/SMC recommended inclusion criteria). ${ }^{8,13-15}$ As well as a two- to three-fold increase in the number of patients eligible for treatment for wet AMD, patients receiving anti-VEGF treatments will require more frequent clinic visits than those treated with vPDT. NICE guidelines for vPDT recommended that patients should be re-evaluated every 3 months after initial treatment. ${ }^{7}$ However, ranibizumab treatment is initiated with a loading phase of three intravitreal injections at 4-week intervals followed by a maintenance phase in which patients are monitored on a monthly basis, and re-dosed if necessary, ${ }^{17}$ whilst pegaptanib is administered once every 6 weeks. ${ }^{18}$ RCOphth have thus predicted that this will translate to a potential six- to nine-fold increase in the wet AMD workload. ${ }^{8}$

Earlier referral guidelines recommended that patients with wet AMD needed to be seen and treated within 2 weeks of diagnosis for effective outcomes from vPDT. ${ }^{8}$ However, in reality, local circumstances have sometimes resulted in substantial variation in the time period between initial diagnosis, referral, and treatment, frequently with significant delays. With the introduction of anti-VEGF therapy, the 2 week recommendation from initial primary presentation to treatment is also supported by RCOphth guidelines. ${ }^{8}$ RCOphth emphasises that all patients should be seen by a retinal specialist within 1 week of primary care presentation and should begin treatment within 1 week of evaluation, and that this should be irrespective of geographical location. ${ }^{8}$ The current RCOphth recommended referral pathway is shown in Figure 1, with adaptations for clinical experience highlighted. Specific recommendations from RCOphth are:

- suspected cases should be referred directly to the nearest AMD centre, eye casualty or eye clinic

- optometrist referrals should go directly to an ophthalmology department rather than a GP to avoid delays

- patient self-referral to an eye casualty/clinic or wet AMD clinic should be encouraged, particularly where there is second eye involvement

Directives issued by the Department of Health and the Welsh Assembly Government require that local health boards and the NHS make funding and resources available to implement NICE technology appraisal guidance, normally within 3 months. ${ }^{13}$ Considering the need for substantially increased resources and, potentially, the restructuring of AMD clinics that may be necessary to commission anti-VEGF therapies, this represents a major challenge. RCOphth has recognised the implications that recent developments are having on practice in the NHS and, in 2007, brought together a number of experts in retinal disease and health commissioning to develop a comprehensive AMD management plan. ${ }^{8}$ The subsequent guide for commissioners and clinicians highlighted the essential role of retinal specialists as team leaders in the new AMD service. ${ }^{8}$ To run an efficient AMD clinic with rapid access, although under logistical constraints, means that business management is now becoming an essential part of the retinal specialist's skill set.

\section{Improving wet AMD clinical capacity and capability}

To commission for wet AMD services in a timely and efficient manner, retinal specialists may now find themselves being required to act as business managers by formulating a business case and calculating budgetary and resource requirements. Their role has evolved into a highly challenging, multifaceted, balancing act between business management, staff management and training, whilst at the same time delivering the best possible standard of patient care. To maximise the likelihood of implementing NICE guidance within recommended time frames, it is prudent to put forward the business case to managers and financial personnel at an early stage and gain support at a top level. In addition, frequent and open communication flow between retinal specialists, hospital management, and the primary care trust (PCT) is important to negotiate costing and to track the progress of wet AMD commissioning.

In light of the need for change to current clinical practice and organisation, a cross-geography, multi-hospital project was initiated (sponsored by 
Wet AMD Referral Pathway Diagram

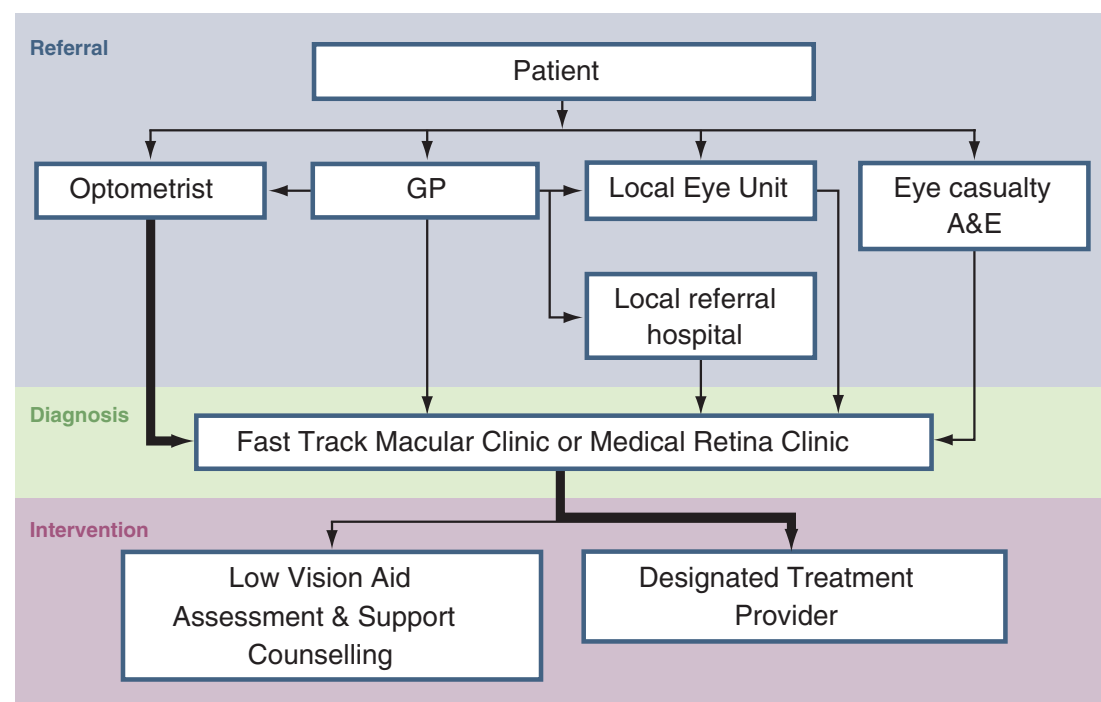

Figure 1 The AMD referral pathway for new patients. Adapted from 'RCOphth commissioning contemporary AMD services: a guide for commissioners and clinicians. ${ }^{8}$ Thick line represents the most common referral pathway, based on experiences at Frimley Park Hospital (FPH) and the Harrogate and District NHS Foundation Trust (HDFT).

Novartis Pharmaceuticals and conducted by ESPRiT Consulting) to identify bottlenecks and establish solutions to good practice in wet AMD clinics. The ultimate aims of the evaluation were to reduce the patient pathway length (where necessary), improve the patient experience and overall level of care, and optimise the cost-effectiveness of anti-VEGF AMD treatment, at each clinic evaluated.

Two of the wet AMD clinics that ESPRiT evaluated were Frimley eye unit at Frimley Park Hospital (FPH) and the ophthalmic clinic at Harrogate District Hospital. The large centre at FPH opened in 2005 and was set up to allow the hospital to treat more patients and reduce waiting times. The centre's modern outpatient department with a catchment area of 1.6 million people for AMD includes 12 consulting rooms, a nurse treatment room, and a laser suite. The Harrogate and District NHS Foundation Trust (HDFT) at Harrogate District Hospital has a smaller ophthalmic outpatient's service and, jointly with York, covers a population of around 800000 for AMD.

Historically, at FPH and HDFT, AMD patients with varying lesion types were frequently referred to the PDT clinic for treatment, but only a subset of these patients was eligible for treatment. The referral pathway was similar to that recommended by RCOphth, but patients were commonly referred from optometrist to ophthalmologist through the GP, causing delays to treatment. Subsequently, a 'fast track' system was set up at FPH and HDFT such that optometrists could refer patients directly via fax to a central co-ordinator. Patients were then assessed within 1 week and treated with vPDT, where appropriate, within 2 weeks. One of the main challenges for the clinics was to re-educate optometrists and local hospital staff, to reduce the number of ineligible patients attending the PDT clinic and improve the speed of referrals. Re-education of primary care staff invariably increases the quantity of referrals, by increasing awareness of wet AMD; however, as a result, the number of false-positive referrals may also increase. At FPH, triannual teaching sessions for optometrists consisting of lectures, practical sessions, and discussion improved the true-positive referral rate to $75 \%$. A critical element in this improvement was continued education about referrals, through constant feedback. In Yorkshire, articles in local newspapers raised the profile of the service and meetings with local optometrists and GPs stressed the importance of fast referrals for patients with suspected wet AMD.

A one-stop rapid access clinic was set up, at both FPH and HDFT, so that eligible patients could arrive, be treated with vPDT rapidly and leave.

The experience gained with PDT clinics was invaluable with the evolution of anti-VEGF therapy. It enabled staff at both hospitals to formulate a business plan for an anti-VEGF clinic and persuade management that more resources were required. A key factor in this process was having previously established positive relationships with 
hospital managers and the finance department. Contacts within the PCT can also be helpful, although this can be more difficult to achieve.

The current patient pathway has evolved from, and is a refinement of, that already described. The anti-VEGF AMD clinics at both FPH and HDFT began as fledgling services alongside the existing PDT service. At HDFT, intravitreal treatments were initially administered in an operating theatre. However, this caused logistical problems and affected other services, such as cataract surgery. A suitable clean room was, therefore, located close to the eye clinic that reduced the movement of patients around the hospital. Both clinics currently run as a one-stop service. One-stop clinics necessitate fewer visits and less travelling for patients, many of whom may have mobility challenges and/or may have to travel a significant distance to attend the clinic. However, it can be difficult to predict the treatment numbers with a

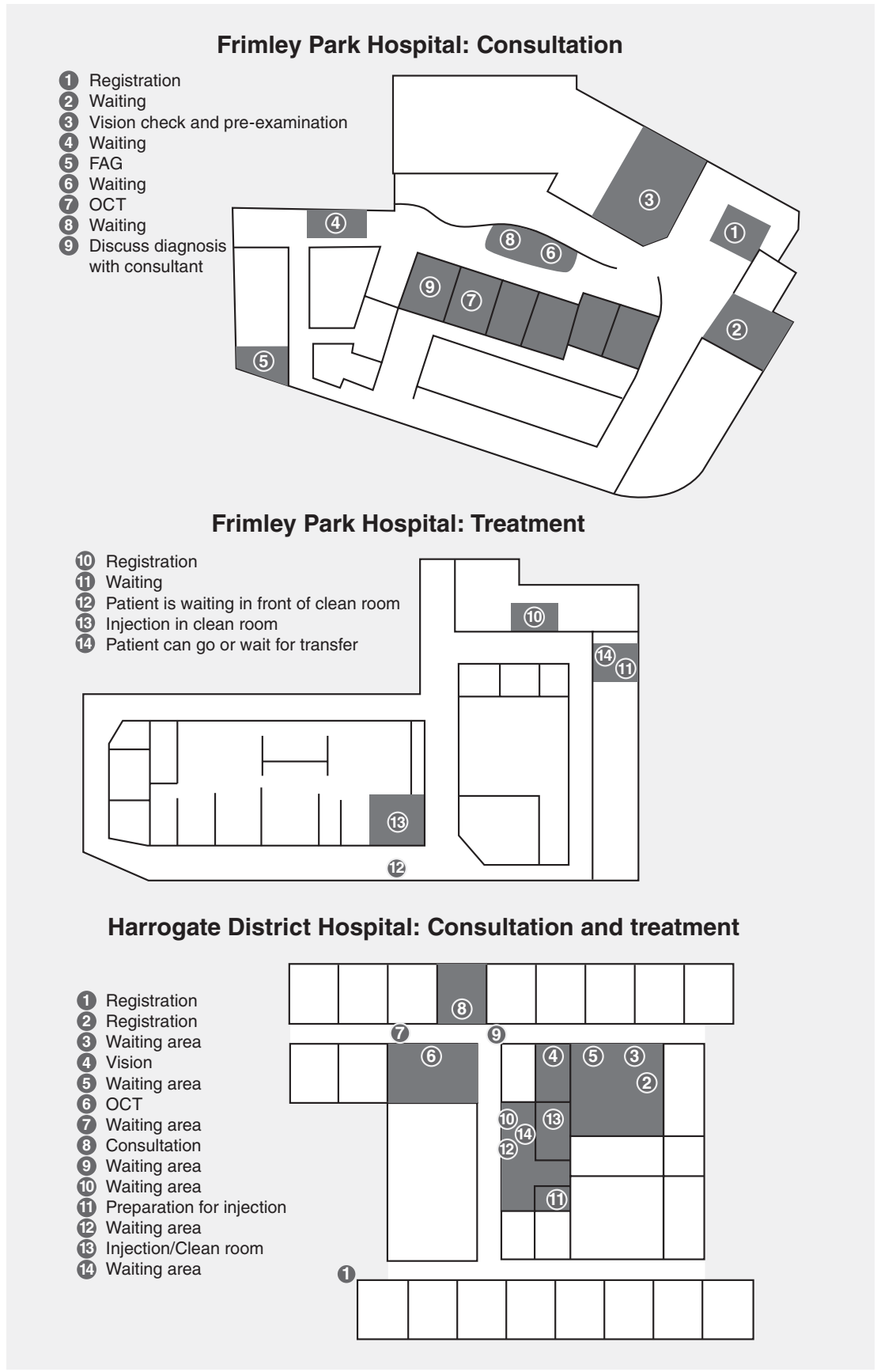

Figure 2 Map of patient flow through clinics at Frimley Park Hospital (FPH) and Harrogate and District NHS Foundation Trust (HDFT) - consultation and treatment. 
one-stop clinic, and may result in longer clinic visits for patients requiring treatment. Earlier experience with a two-stop clinic at HDFT showed that such a system is less practical than a one-stop regime, but may make better use of theatre time when an operating theatre is used for intravitreal injections. With an accurate and evidence-based business plan as the foundation, both the
FPH and HDFT AMD clinics have expanded dramatically while retaining an efficient patient service. The service at HDFT has evolved from a once-fortnightly clinic treating only the second eye, to four clinics each week, now with PCT backing to treat any eye. There are currently 170 wet AMD patients being assessed and/or treated at HDFT. The number of clinics at FPH has now

\section{Step 1 - Develop a business case}

A strong business case supported with patient numbers and financial figures, will ensure that hospital managers are well-informed and supportive of the requirements. Critical success factors to developing an accurate and successful business case include:

- A realistic awareness of likely referral numbers and knowledge of where these are coming from

- Early engagement of managers

- Consideration and justification for adequate resources

Space - The service requires a large amount of space to contain; waiting room, vision alleys, camera room, OCT room, clinic rooms, preparation area and treatment (clean) room. Ideally the clean room should be located in close proximity to the clinic rooms

Staffing - Approximately 1 doctor per 12 patients per clinic (depending on facilities), and at least 3 qualified and 1-2 non-qualified nursing staff are required. Additionally, there should be adequate administrative and secretarial staff, and pharmacy time

Time - It is important not to underestimate the time it takes to set up and run an anti-VEGF AMD clinic

Equipment - Essential equipment includes; logMAR vision charts, OCT machines and disposable treatment packs IT - A paperless system is ideal with an integrated system such as Medisoft Ophthalmology (Medisoft Limited, Leeds, UK)

\section{Step 2 - Establish commissioning}

- Negotiation of realistic costing with the PCT. Although each hospital may negotiate cost with their PCT on an individual basis, it is essential to negotiate a realistic tariff

- Development of a streamlined billing mechanism

- Regular communication with stakeholders. Keeping all stakeholders involved at every step will help to achieve buyin from those involved and contribute to fast and efficient implementation

\section{Step 3 - Educate Healthcare Professionals and internal personnel}

It is helpful to include all internal personnel who will see the patient in training and education, so that they are fully aware of relevant issues and the importance of dealing with patients rapidly and efficiently. Including:

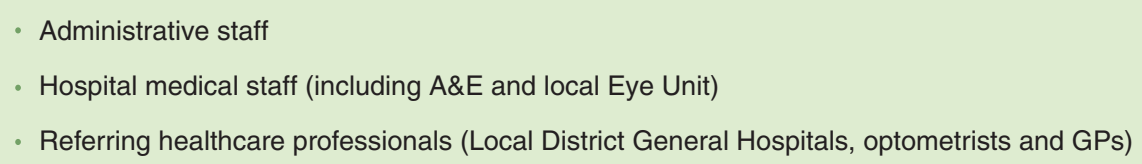

Local educational meetings can effectively reduce the number of false-positive referrals. However, it is worth noting that education of referring staff often leads to an increased awareness of the disease and hence an increase in the overall referral numbers (and initially, in the number of false-positive cases). To refine this process, continuous feedback to optometrists and GPs relating to their referred patients is important to help further develop education and keep them abreast of the current condition of the patient.

\section{Step 4 - Continually review and refine the process}

Continuous review of the business case and processes is necessary, to ensure optimum running of the clinic, particularly in the early stages. Small, seemingly insignificant, alterations to the process (for example regular staff meetings) can have a substantial impact on the patient experience.

Figure 3 A step-by-step guide to setting up and running an efficient wet AMD clinic. 
increased to five clinics per week, and is currently assessing and treating around 320 patients. The numbers of patients at both FPH and HDFT are continuing to increase, in line with an estimated incidence of the disease of 45 per 100000 population. ${ }^{8}$

Formal external evaluations rated patient examination and treatment processes highly, and the interface with primary care at both FPH and HDFT. Areas identified for improvement were administration, data management and IT. For example, HDFT only had one logMAR vision chart and both clinics only had one optical coherence tomography (OCT) machine. Further recommendations included that staff at the large FPH clinic should hold meetings to discuss process issues every 6-8 weeks. On the basis of these recommendations, the services at both clinics have benefited. More logMAR charts were ordered at HDFT, and both clinics ordered second OCT machines, increased staffing and administrative support, and put an integrated IT system in place. Regular staff meetings at FPH have helped to further optimise processes and ensure a smooth-running service. The evaluation process also mapped the patient journey through the clinic at FPH and HDFT (Figure 2). One of the key recommendations, for both centres, was that a clean room for treatment should be located close to the patient assessment room to reduce patient movement through the hospital.

\section{A step-by-step guide to setting up and running an efficient wet AMD clinic}

On the basis of experiences at FPH and HDFT, we have identified a number of critical success factors for an efficient anti-VEGF AMD clinic. We have amalgamated our key learnings to propose the following practical guide to setting up and efficiently running a clinic (Figure 3). Where there is currently no existing service available, it can be expected that an initial backlog of patients will present once a new service is put in place, and this should be borne in mind.

\section{Summary}

Wet AMD is a degenerative eye disease that requires rapid referral and treatment to prevent loss of detailed central vision. ${ }^{1,9}$ Anti-VEGF treatment guidance from NICE and the SMC have led to an increased number of patients eligible for wet AMD treatment, and also the number of clinic visits per patient, have substantially increased compared with vPDT. ${ }^{8}$ Clinics offering a wet AMD treatment service will be required to adapt swiftly to be able to implement the guidelines within a reasonable time frame, but this will dramatically impact resources and procedures. The need for change has highlighted an evolving role for retinal specialists, with business management becoming an essential part of the skill repertoire. ${ }^{8}$ Earlier experience gained from PDT clinics can provide a valuable foundation on which to build a robust business case for commissioning antiVEGF treatment. However, the contemporary wet AMD clinic is a constantly evolving process, and modifications and refinement of processes are expected as experiences develop.

\section{Disclosure}

G Walters has received lecture fees and consulting fees from Novartis Pharmaceuticals Ltd (UK). G Menon has received lecture fees and grant support from Novartis Pharmaceuticals and grant support from Pfizer.

\section{Acknowledgements}

This article was supported by Novartis Pharmaceuticals Limited (UK). The company has had the opportunity to comment on the medical content and accuracy of the article; however, final editorial content resides with the author and the journal. The authors thank Sue Harris from Huntsworth Health, who provided medical writing support on behalf of Novartis Pharmaceuticals.

\section{References}

1 Wong T, Chakravarthy U, Klein R, Mitchell P, Zlateva G, Buggage $\mathrm{R}$ et al. The natural history and prognosis of neovascular age-related macular degeneration. Ophthalmology 2008; 115: 116-126.

2 Schmidt-Erfurth UM, Elsner H, Terai N, Benecke A, Dahmen G, Michels SM. Effects of verteporfin therapy on central visual field function. Ophthalmology 2004; 111: 931-939.

3 Bunce C, Wormald R. Causes of blind certifications in England and Wales: April 1999-March 2000. Eye 2008; 22: 905-911.

4 Ferris III FL, Fine SL, Hyman L. Age-related macular degeneration and blindness due to neovascular maculopathy. Arch Ophthalmol 1984; 102: 1640-1642.

5 Jager RD, Mieler WF, Miller JW. Age-related macular degeneration. $N$ Engl J Med 2008; 358: 2606-2617.

6 Nowak JZ. Age-related macular degeneration (AMD): pathogenesis and therapy. Pharmacol Rep 2006; 58: 353-363.

7 TA68 Macular degeneration (age related) - photodynamic therapy: Guidance. 2003. Available at http://www.nice. org.uk/nicemedia/pdf/68_PDTGuidance.pdf (accessed on 1 February 2009).

8 RCOphth commissioning contemporary AMD services: a guide for commissioners and clinicians. 2007. Available at http:// www.rcophth.ac.uk/docs/publications/published-guidelines/ CommissionContempAMDServicesV3Final.pdf (accessed on 1 February 2009). 
9 Rosenfeld PJ, Brown DM, Heier JS, Boyer DS, Kaiser PK, Chung CY et al, MARINA Study Group. Ranibizumab for neovascular age-related macular degeneration. $N$ Engl J Med 2006; 355: 1419-1431.

10 Brown DM, Kaiser PK, Michels M, Soubrane G, Heier JS, Kim RY et al, ANCHOR Study Group. Ranibizumab versus verteporfin for neovascular age-related macular degeneration. N Engl J Med 2006; 355: 1432-1444.

11 Regillo CD, Brown DM, Abraham P, Yue H, Ianchulev T, Schneider S et al, PIER Study Group. Randomized, double-masked, sham-controlled trial of ranibizumab for neovascular age-related macular degeneration: PIER Study year 1. Am J Ophthalmol 2008; 145: 239-248.

12 Gragoudas ES, Adamis AP, Cunningham Jr ET, Feinsod M, Guyer DR, VEGF Inhibition Study in Ocular

Neovascularization Clinical Trial Group. Pegaptanib for neovascular age-related macular degeneration. $N$ Engl J Med 2004; 351: 2805-2816.

13 Macular degeneration (age-related)-ranibizumab and pegaptanib: Final appraisal determination. 2008. Available at http://www.nice.org.uk/nicemedia/pdf/

TA155guidance.pdf (accessed on 1 February 2009).
14 Ranibizumab $10 \mathrm{mg} / \mathrm{ml}$ solution for intravitreal injection (No. 381/07). Available at http://www.scottishmedicines. org.uk/smc/files/ranibizumab_10mgmlsolution intravitrealinjection_Lucentis_38107.pdf (accessed on 1 February 2009).

15 Pegaptanib $0.3 \mathrm{mg}$ solution for intravitreal injection (No. 290/06). Available at http://www.scottishmedicines. org.uk/smc/files/pegaptanib_Macugen_290_06.pdf (accessed on 1 February 2009)

16 The Royal College of Ophthalmologists. Ranibizumab: the clinician's guide to commencing, continuing and discontinuing treatment. Available at http:// www.rcophth.ac.uk/docs/scientific/Ranibizumab_June_ 2008.pdf (accessed on 1 February 2009).

17 Lucentis: summary of product characteristics. Available at http://www.emea.europa.eu/humandocs/ PDFs/EPAR/lucentis/H-715-PI-en.pdf (accessed on 1 February 2009).

18 Macugen: summary of product characteristics. Available at http://www.emea.europa.eu/humandocs/ PDFs/EPAR/macugen/H-620-PI-en.pdf (accessed on 1 August 2008). 